Mappemonde

Revue trimestrielle sur l'image géographique et les formes du territoire

122 | 2017

Varia

\title{
Iran, une société en ébullition
}

Paris : HD Ateliers Henry Dougier, 2017

Clarisse Didelon-Loiseau

\section{(2) OpenEdition}

Journals

Édition électronique

URL : http://journals.openedition.org/mappemonde/568

DOI : 10.4000/mappemonde.568

ISSN : $1769-7298$

Éditeur

UMR ESPACE

\section{Référence électronique}

Clarisse Didelon-Loiseau, «Iran, une société en ébullition », Mappemonde [En ligne], 122 | 2017, mis en ligne le 08 décembre 2017, consulté le 24 septembre 2020. URL : http://journals.openedition.org/ mappemonde/568; DOI : https://doi.org/10.4000/mappemonde.568

Ce document a été généré automatiquement le 24 septembre 2020

\section{(c) (i) (8)}

La revue Mappemonde est mise à disposition selon les termes de la Licence Creative Commons Attribution - Pas d'Utilisation Commerciale - Partage dans les Mêmes Conditions 4.0 International. 


\section{Iran, une société en ébullition}

Paris : HD Ateliers Henry Dougier, 2017

\section{Clarisse Didelon-Loiseau}

\section{RÉFÉRENCE}

Iran, une société en ébullition, mars 2017, Paris : HD Ateliers Henry Dougier, collection MAPPE. 
1 La collection « Mappe » est une collection de «livres pliés» qui rassemble «les analyses des meilleurs experts internationaux » pour mettre "en scène les basculements du monde ». Le volume sur l'Iran est le $9^{\text {e }}$ de la collection, mais seulement le $3^{\mathrm{e}}$ volume sur un pays après la Chine et la Russie. En l'absence d'un nom de coordinateur du volume, il est probable que les textes aient été assemblés et édités par la coordination éditoriale ; il ne s'agit donc pas réellement d'un «ouvrage collectif», mais d'une collection de 8 courts articles de chercheurs spécialistes de l'Iran plus «une carte géante» qui, selon la présentation de la collection, est censée proposer «une découverte visuelle


spectaculaire des enjeux » de la région. Le principe du livre plié est que l'on découvre les articles au fur et à mesure que l'on déplie le livre (et que le format s'agrandit); ce dépliage organise les 4 rubriques, trois qui concernent les textes et une qui est réservée à la carte géante.

\section{Rubrique « L'œil de l'expert », première double page}

2 Il s'agit d'un entretien avec Bernard Hourcade, ayant pour titre «Le régime iranien, un équilibre fragile », qui est assez pédagogique dans sa construction et son contenu et qui balaie les grands thèmes qui sont ensuite abordés dans le reste du volume.

\section{Rubrique « Un territoire, une géographie », quadruple page}

3 Cette rubrique est composée de deux articles. Le premier est écrit par Yann Richard, un spécialiste de "sociologie religieuse du chiisme moderne" et historien de l'Iran. Il porte sur la prise de pouvoir de Khomeyni. L'article est court, assez allusif et présente une succession de faits non expliqués. Par exemple, «L'arrivée de Khomeny en France, le 6 octobre 1978, donne à ce clerc intransigeant une stature de grand sage ». On ne sait pas pourquoi il vient en France ni pourquoi ce séjour lui donne une telle stature.

Le second article est un entretien avec Amir Nikpey (chercheur iranien), sur la sociologie des pratiques religieuses et les religions dans la société iranienne. Le contenu est à nouveau très allusif, peu explicité. Des contradictions dans le texte soulignent peut-être un manque de soin dans l'édition (au sein du même article on trouve ainsi : paragraphe 2: "L'Iran est très divers sur le plan des pratiques religieuses ». Paragraphe 5: «La religion en Iran est donc plurielle dans la croyance, mais pas au niveau des pratiques »). La carte jointe à l'article est une carte historique qui positionne 
les « centres du pouvoir achéménide » et les extensions de différents empires ayant eu pour « centre » la Perse. Elle n'a aucun rapport avec le texte, même si elle est soignée. Elle est réalisée "en partenariat » avec la revue Moyen-Orient, ce qui semble indiquer qu'elle a déjà été éditée (dans le n 32, en 2016 selon les indications portées sur la carte) et donc qu'il ne s'agit pas d'un document original.

5 Cette rubrique « Un territoire, une géographie » est donc composée de deux textes de teneur plutôt historique et portant sur la sociologie des religions dans la société iranienne. En dépit du titre, il n'y a donc pas de géographie.

\section{Rubrique « Un territoire, des enjeux », octuple page}

6 Cette rubrique est composée de 5 articles accompagnés d'un encadré " pour en savoir plus » qui liste des essais sur l'Iran, ainsi que quelques romans, BD et films dont les auteurs sont iraniens.

7 Le premier article, de Mohammad-Reza Djalili et Thierry Kellner a pour titre : «Iran/ Arabie Saoudite: une rivalité multiforme » et présente un historique des relations entre Iran et Arabie Saoudite. Il est accompagné d'une photographie de Bandar Abbas, dont «la position stratégique sur le détroit d'Ormuz» évoquée en légende de l'illustration n'est pas expliquée et n'est pas évoquée dans le texte.

8 Le deuxième article «Être iranienne : une lutte pour l'égalité et la liberté » est celui d'une journaliste correspondante du Monde en Iran, Gahzal Golshiri. Elle évoque une question sensible pour le public occidental, mais en le traitant de manière quasi anecdotique : après un très court paragraphe sur les désillusions électorales des jeunes Iraniens et le statut juridique des femmes, elle dresse le portrait de 4 femmes trentenaires engagées dans la vie civique iranienne. L'article ne dit rien de la manière dont sont reçues ces initiatives par la société en général et les autres femmes en particulier et il se termine par une affirmation (non étayée) qui est à ranger dans la catégorie des «vœux pieux»: «ce sont ces femmes qui conduiront enfin l'Iran vers davantage d'égalité et de liberté ».

9 Le troisième article, de Clément Therme, porte sur le processus de «normalisation des relations économiques » en cours en évoquant les choix qui s'offrent à l'Iran après l'accord sur le nucléaire de 2015, et les réticences des sociétés occidentales. Encore une fois, la majorité de l'article comprend des passages beaucoup trop allusifs qui ne sont d'aucune utilité sinon factuelle, puisqu'on peine à percevoir les enjeux de ce qui est décrit.

10 Enfin, deux articles portent sur deux facettes culturelles appréciées des intellectuels occidentaux, en particulier français, la littérature et le cinéma iranien. L'article de Laetitia Nanquette porte sur « La création littéraire d'un pays s'ouvrant au monde » et présente un inventaire rapide de livres dont on peine à saisir la tendance et les implications. L'article évoque rapidement la diversité des formes de création littéraire et la place des femmes dans celle-ci, mais ne livre pas vraiment d'analyse. L'article d'Asal Bagheri qui évoque "Le cinéma d'Asghar Farhadi et la société iranienne" propose une analyse rapide, mais une analyse, des films d'Asghar Farhadi et voit dans ce cinéaste le représentant malgré lui d'une idéologie patriarcale et misogyne dominante du fait des scènes de «violences physiques contre les personnages féminins » présentes dans ces films. 


\section{Rubrique « Un territoire, une carte »} $\left(100^{*} 68 \mathrm{~cm}\right)$ intitulée "L'Iran, une puissance géographique et économique ». Elle est composée d'une grande carte et de sa légende, de deux encarts cartographiques et d'un encart factuel. sien. Elle est construite sur un fond de carte de géographie physique réalisé dans un grisé discret et accompagnée du réseau hydrographique. Elle comprend différents symboles qu'éclaire la légende. Celle-ci est organisée en grands thèmes : géographie physique (lacs, etc..), «économie» (zones franches, ZES), "conflits et tensions » (symboles en étoile localisés sur les autres pays + zones maritimes contestées); puis «tourisme », " pétrole» et " gaz » ainsi que la population des principales villes. Le choix des thèmes représentés ne semble que peu répondre au titre problématisé de la carte et ne permet pas de comprendre en quoi l'Iran est une puissance (ni d'ailleurs ce qu'est une puissance " géographique »). La carte considérée dans son ensemble permet juste de comprendre que la majorité de la population et de certaines activités sont plutôt localisées dans le nord-ouest du pays.

13 Les deux encarts cartographiques représentent d'une part «la diversité linguistique en 2014 ", ce qui semble sans rapport avec le titre de la composition cartographique et d'autre part une carte à l'échelle du Moyen-Orient qui présente «l'environnement géostratégique et nucléaire de l'Iran en 2017 » à l'aide d'une information qualitative. Dans la légende il manque la signification des flèches jaunes et grises qui partent respectivement d'Iran et d'Arabie Saoudite vers les autres pays (parfois les mêmes: Irak, Yémen, Afghanistan). À propos de cette carte, on se demande pourquoi elle n'a pas été mise en regard de l'article de Mohammad-Resa Djalili et Thierry Kellner, avec lequel elle aurait semblé pertinente.

Enfin, un encart présente des informations factuelles sur le territoire (capitale, superficie, monnaie, etc.), la population en 2015 (densité, ethnies, confessions religieuses) et l'économie en 2015 (PIB, chômage, ressources énergétiques).

En ce qui concerne l'ensemble de la composition cartographique, tout est écrit trop petit pour qu'on puisse regarder l'ensemble de loin (et donc accrocher la carte au mur), mais de près, le format déplié n'est pas maniable et la plus grande des cartes se laisse difficilement percevoir dans son ensemble. Cette composition cartographique ne semble donc pas atteindre le but affiché de la collection (proposer « une découverte visuelle spectaculaire des enjeux" de la région), puisque la seule chose qui soit réellement spectaculaire ici c'est la taille de la composition. En ce qui concerne les enjeux, il est malaisé de les saisir, d'autant que les thèmes évoqués sur la carte (tourisme, population, gaz, pétrole...) ne le sont pas dans les articles.

Selon les indications indiquées sur le fascicule, la carte est accompagnée d'un commentaire en vidéo sur "YouTube ». L'ouvrage ne fournit aucune indication sur la manière dont on peut accéder à la vidéo. À l'heure des $Q R$ code c'est dommage... Si on entreprend de rechercher la vidéo sur « YouTube », on n'est pas au bout de ses peines.

Mappemonde, 122 | 2017 

plein de promesses en ce qui concerne la carte centrale, il se révèle finalement peu maniable et d'autant plus décevant que la carte est, au final, peu utile, malgré la qualité formelle dont elle fait preuve. En ce qui concerne le contenu, on se trouve face à un assemblage relativement hétéroclite d'images, de textes et de cartes. Les documents visuels ont peu de rapport avec le contenu textuel. Le contenu des articles des différents experts est parfois redondant. L'ensemble apparait peu utile aux spécialistes qui connaitront plus de choses que ce qu'il y a dans le texte et peu utile également aux non-spécialistes qui ne comprendront pas réellement les ressorts de l'enchainement des évènements puisque la majorité des articles adoptent une perspective historique. On peine de fait à identifier le public cible de cette collection et de ce volume, dont l'intérêt consiste peut-être seulement à donner envie aux curieux d'en savoir plus.

\section{AUTEURS}

\section{CLARISSE DIDELON-LOISEAU}

Université Paris 1 